\title{
Exosomes: the future of biomarkers in medicine
}

Exosomes are nanovesicles secreted into the extracellular environment upon internal vesicle fusion with the plasma membrane. The molecular content of exosomes is a fingerprint of the releasing cell type and of its status. For this reason, and because they are released in easily accessible body fluids such as blood and urine, they represent a precious biomedical tool. A growing body of evidence suggests that exosomes may be used as biomarkers for the diagnosis and prognosis of malignant tumors. This article focuses on the exploitation of exosomes as diagnostic tools for human tumors and discusses possible applications of the same strategies to other pathologies, such as neurodegenerative diseases.

\section{KEYWORDS: biomarkers exosomes nanotheranostics nanovesicles neurodegenerative diseases prion diseases tumor diagnosis}

Exosomes are nanovesicles of 50-100 nm diameter that are released from most viable cells. Current knowledge suggests that exosomes correspond to the intraluminal vesicles of endosomal multivesicular bodies (MVBs), released in the extracellular environment after MVB fusion with the plasma membrane. They form by membrane invagination of late endosomes, resulting in vesicles that contain cytosolic components and expose the extracellular domain of some plasma membrane receptors at their surface $[1,2]$. Despite this well-established hypothesis, the contribution of other MVB-independent mechanisms to the exosome formation process cannot currently be ruled out. Because of their cellular origin, exosomes bear specific protein markers of the endosomal pathway, such as tetraspanins (CD63, CD9 and CD81), heat shock proteins (HSP70) and proteins from the Rab family, Tsg101 and Alix, which are not found in other types of vesicles of similar size [3,4]. Ectosomes, for example, are formed upon plasma membrane shedding and contain markers such as integrins and P-selectin, which are not related to the endosomal/exosomal pathway and are CD63 negative [5]. Similarly, apoptotic particles can be distinguished as they selectively contain histones [1].

Exosomes were first identified in the harvested media of reticulocyte cell cultures as microvesicles containing membrane proteins, including the transferrin receptor [6]. Since then, several cell types have been described to release exosomes into the extracellular environment. Hematopoietic cells (B cells, T cells, dendritic cells, mast cells and platelets), intestinal epithelial cells,
Schwann cells, neuronal cells, adipocytes, fibroblasts (NIH3T3) and tumor cells have all been shown to secrete MVB internal vesicles [4].

The physiological meaning of MVB internal particle release into the extracellular space is still a matter of debate. They were originally suggested to be involved in the removal of unnecessary molecules that are poorly degraded by the lysosomal system [7]. Nevertheless, as research progresses, the exosomal functions appear to be far more complex. Platelets secrete exosomes that are able to regulate the coagulation response [5]. Immune system cells, such as B cells and dendritic cells, mediate MHC-dependent immune responses upon nanovesicle secretion [8,9]. For this purpose, vesicles are provided with specific adhesion molecules for narrow targeting of recipient cells [10]. Dendritic cell-derived exosomes, eluted from a variety of different tumors, eradicate and suppress the growth of murine tumors in a T-cell-dependent manner [11]. Interestingly, as antigen-presenting vesicles, exosomes are more immunogenic than soluble molecules and their use as safe vaccines is currently being investigated [12].

Further fascinating studies have shown that exosomes secreted by glioblastoma and mast cells can carry mRNAs encoding up to 1300 genes that are delivered and properly translated into target cells. The vesicle-associated RNA is called exosomal shuttle RNA and includes small RNA species, such as miRNAs [13,14]. Exosomal miRNAs are functional in target cells where they produce effective gene silencing [15].

All these remarkable findings suggest that exosomes are not only cell 'cleaners', but
Francesca Properzi ${ }^{1}$,

Mariantonia Logozzi ${ }^{2}$

\& Stefano Fais ${ }^{* 2}$

¿Department of Cell Biology

\& Neurosciences, Istituto Superiore di

Sanità, Rome, Italy

${ }^{2}$ Department of Therapeutic Research

\& Medicines Evaluation, Istituto

Superiore di Sanità, (National Institute

of Health), Viale Regina Elena, 299,

o0161 Rome, Italy

*Author for correspondence:

Tel.: +3906 49903195

Fax: +390649902436

stefano.fais@iss.it 
operating cell shuttles playing a crucial role in cell-to-cell communication. They probably also work as highly specialized functional extensions of cells, increasing their area of action. They are largely released in biological fluids, such as plasma, urine, cerebrospinal fluid, epididymal fluid, amniotic fluid, malignant and pleural effusions of ascites, bronchoalveolar lavage fluid, synovial fluid and breast milk, suggesting a role in bridging the exchange of information across distant body compartments [16].

Interestingly, the molecular contents of the exosomes that are secreted in body fluids have proven to be highly specific and a precious biomedical tool [17-19]. Some recent data suggest that exosome purification may represent a more informative diagnostic tool than whole body fluid. Cells located towards the drainage system, such as kidney tubular cells, provide exosomal markers that are not detectable in whole urine [17]. Urine exosomal preparations from prostate cancer patients indeed contain mRNA of PCA3 and TMPRSS2, a product resulting from an ERG fusion chromosomal rearrangement [18]. Similarly, in urine exosomes, diagnostic and prognostic markers of renal ischemia/reperfusion injury or antidiuretic hormone action, such as aquaporin-1 and -2 , have been found $[19,20]$. All these molecules are not easily detected in whole urine.

The power of nanovesicles as biomarkers relies on the enrichment of highly selected markers during exosomal sorting, which otherwise constitute only a very small proportion (less than $0.01 \%$ ) of the total proteome of body fluids. The enrichment of diagnostic markers at the exosomal source, due to cargo sorting into exosomes, helps the discovery of relatively lowly expressed biomarkers that normally would go undetected. In healthy human-circulating exosomes, approximately 66 proteins were identified, most of which were involved in vesicular trafficking pathways [21]. The exosome biomarker strategy exemplifies the concept of 'less being more', as the small number of proteins and RNAs give very insightful information that is specifically associated with pathological conditions.

Through an adapted ELISA test allowing the detection, characterization and quantification of exosomes [22], it has been shown that tumor patients have significantly increased plasmatic levels of exosomes expressing CAV1 compared with the plasma of healthy donors. Moreover, plasmatic exosomes deliver lung tumor-associated miRNAs, suggesting that circulating exosomal miRNAs are representative of those expressed in the tumors and opening a perspective for early detection of the disease [23]. Preliminary studies have shown that salivary exosome protein and miRNA content can be potentially useful to detect disease conditions, such as Sjogren's syndrome [24,25], and exosomes from amniotic fluids could provide a potent tool for prenatal diagnosis [26].

Exosome profiling could provide tailored information of individual patients and may be crucial for a precise prognosis or a differential diagnosis. The quantity of secretomes converging into body fluids, and the variety of cell types contributing to it, increases the noise of fluidbased analysis systems [27]. It is a novel idea that exosomal biomarker changes could be indicative of more than one condition. It is likely that plasma exosomes are released by different cell sources, from both blood cells and tissues that are then filtered by endothelial cells and large fenestrated organs such as the placenta and the liver. Therefore, isolation of cell-specific exosomes by immunoprecipitation could be a successful method to trace differential changes, thus discriminating the cell lineages that produce them. Plasma exosomes of hepatic origin can be isolated using antibodies that are specific to the liver marker carbamoyl phosphate synthetase 1 [28]. In cell cultures, A33 affinity isolation of colorectal cancer exosomes [28] and CD45 isolation of Jurkat T-cell line exosomes has yielded very specific cell-based signatures [29], and is currently being validated in the plasma of patients.

Thus, body fluid exosomes constitute a source of multiple markers that provide clinically useful information. An initial screening in conditioned media from cultured cells can be used to build a predictive model of deregulated pathways represented in exosomes. Models can then be translated in vivo into biological fluid sampling from a large patient cohort.

\section{Exosome-based strategies for the diagnosis of tumors}

Exosome-associated diagnostic molecules include exosomal proteins and RNAs (TABLE 1). A number of publications have described the association of exosomal markers with several cancer types; nevertheless, only a few exosomebased diagnostic assays are currently available for clinical use. Recently, an exosome-based tumor diagnostic assay called ExoTESTTM (HansaBioMed, Estonia) was developed in our laboratories. ExoTEST is a sandwich ELISA developed to capture and quantify exosomes in human plasma and other body fluids, as well 
as in cell culture supernatants. In this method, proteins of exosomes such as CD63 and Rab5 b, and a tumor-associated marker (e.g., CAV1) are exploited for immunoisolation of nanovesicles [22]. The accuracy of the novel method was established by western blot and flow cytometry analysis. In clinical studies it was observed by using ExoTEST that $\mathrm{CD} 3^{+}$and $\mathrm{CAV1} 1^{+}$plasma exosome levels were significantly higher in melanoma patients compared with healthy donors. Specifically, the detection of $\mathrm{CD}^{+} 3^{+}$plasma exosomes by ExoTEST had a sensitivity of $43 \%$, while a higher sensitivity of $68 \%$ was estimated for the detection of $\mathrm{CAV}^{+}$plasma exosomes. Furthermore, in melanoma patients, plasma exosomes expressing $\mathrm{CAV}^{+}$were significantly higher when compared with plasma exosomes expressing $\mathrm{CD}^{+} 3^{+}$. Interestingly, the values of CD63 and CAV1 were compared with the serum level of LDH. Although the majority of patients were classified as stage III and IV (86/90), only six of them showed significantly higher serum levels of LDH, with no apparent correlation with staging. Other patients presented very heterogeneous and slightly above average levels of LDH. By contrast, the optical density values of CAV1 and CD63 were distributed through a scale that was compatible with exosomal quantities (measured as values of CAV1) and tumor staging. Consistently with these clinical findings, the ExoTEST allowed sensitive detection and quantification of exosomes purified from human tumor cell culture supernatant and plasma from severe combined immunodeficiency mice engrafted with human melanoma. Interestingly, plasma levels of exosomes in melanomaengrafted severe combined immunodeficiency mice correlated to tumor size, consistent with the finding.

Together, these data suggest that ExoTEST can be used for the determination and quantification of circulating exosomes, providing potential new sources of clinical biomarkers for screening tests, diagnosis and follow-up of cancer patients, and also patients with other diseases. In fact, the ExoTEST approach circumvents the common pitfalls of standard methods employed for detection of low abundant peripheral biomarkers. The advantages of this technology over alternative methods are numerous: it is noninvasive, user friendly, rapid, affordable, specific, quantitative, versatile (easily extendable to other markers/conditions), requires small amounts of sample, has multiple readouts and allows follow-up applications. ExoTEST provides the possibility of screening multiple markers on one

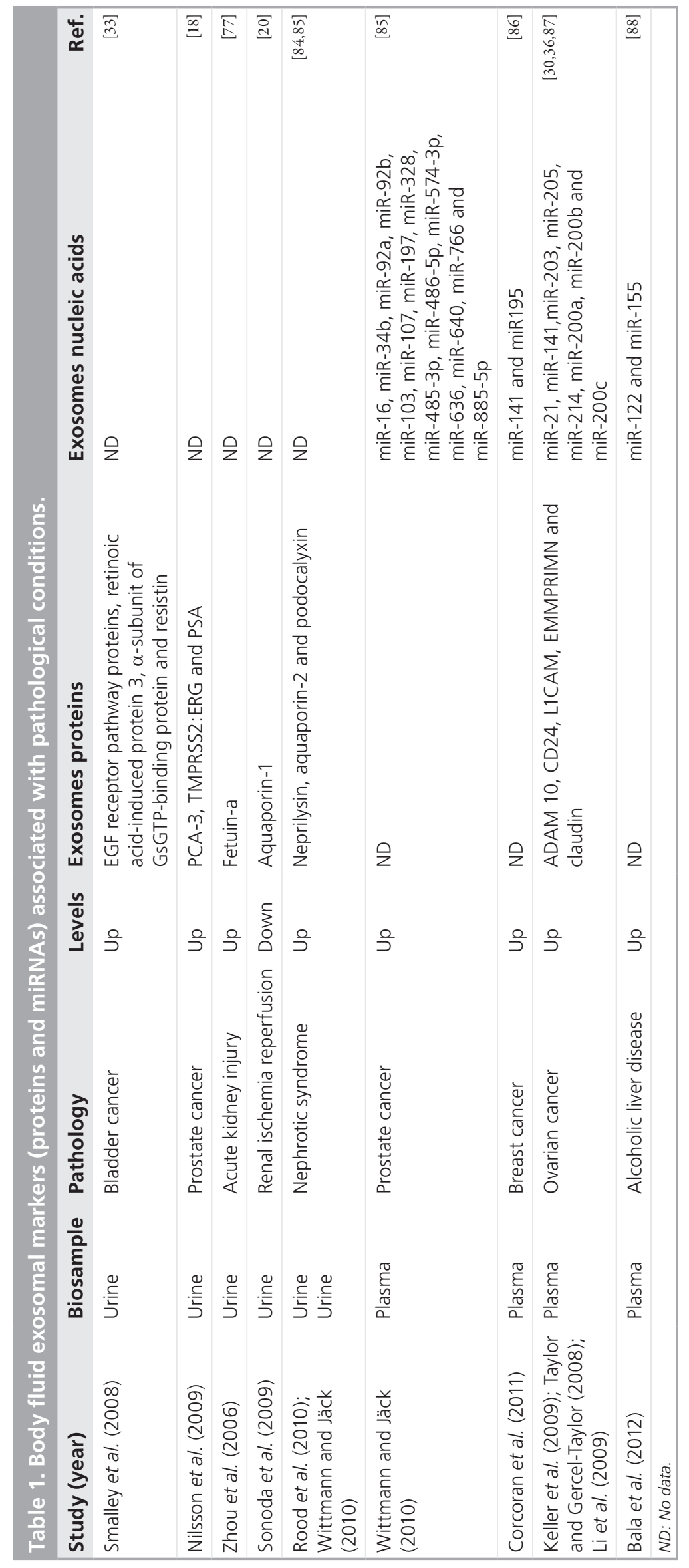


sample, defining conditions with similar clinical presentation and overlap of molecular pathology. Overall, this method will enable the use of already established tumor-associated molecules as peripheral markers, as well as identify and validate novel diagnostic targets, predicted by preliminary tumor screening or discovered by large-scale profiling. By screening a larger number of cancer patients, it might be possible to define better marker profiles for diagnosis and prognosis, as well as develop easy and robust high-throughput assays for routine applications. An additional advantage of both basic and multiplex ExoTEST is the isolation of exosomes from unfractionated plasma, avoiding expensive and time-consuming ultracentrifugation procedures. In addition, the levels of ExoTEST sensitivity could be improved further by including a pool of different markers for better defining circulating exosomes released by the disease site.

Independently from the novel ExoTEST approach, it was found that exosomes that express tumor progression-related proteins, such as L1 cell adhesion molecule, CD24 and the extracellular matrix metalloproteinase inducer, are at higher levels in ovarian tumor patients than in healthy subjects, in both malignant ascites and serum [30].

Exosomes of the prostate epithelium that are released into semen or urine (prosteasome) also contain important molecular information related to certain types of tumors. For example, analysis of urine exosomes of prostate cancer patients identified tumor biomarkers such as prostatespecific antigen and PCA3 [18,31]. Patient urine exosomes and PC-3 cell culture-derived vesicles both also have an increased level of $\mathrm{D}$-catenin [32]. In addition, in some cases of bladder cancer, eight upregulated exosomal proteins were identified. Interestingly, it was found that they largely relate to the EGF receptor signaling mechanism [33]. These findings show that exosomes not only contain precious biomarkers but their profiling can be used to identify specific altered pathways.

ExoTEST is currently being implemented for the high-throughput detection of exosomal RNAs. The persistence of circulating RNA in the blood of cancer patients has recently gained importance as several reports proved the presence of exosome-shuttled RNA, and are thus concentrated and protected from degradation by ribonucleases of human plasma [34]. Incorporating ExoTEST technology into a platform for exosome-associated mRNA analysis is expected to enable detection and quantification of plasma RNAs of well-defined tumor origin, providing highly sensitive and specific assessment while avoiding some of the 'noise' that hampers quantitative real-time PCR and microarray analysis of whole body fluids. Besides, exosomes can be enriched in mRNAs and miRNAs, which are hardly detectable in the parent tissue where their signal is covered by the presence of a higher number of molecules. Thus, they can be the source for identification of novel disease-associated markers. For example, miR-195 levels are known to be higher in breast tumor patients. The upregulation of this biomarker was also confirmed on the plasma exosome. The same authors described that miR-195 and let-7a levels decreased upon tumor removal, showing a possible application as a prognostic biomarker as well. Other authors showed that glioblastoma-derived exosomes carry a diagnostic set of miRNAs [35] and that plasma exosomes of prostate cancer patients contained higher levels of miR-145 [31]. In ovarian cancer, upregulation of a number of miRNAs was found in blood exosomes [36] and exosomal miRNA levels are also significantly higher in patients with lung adenocarcinoma and lymphoma [37].

A current limitation of exosome diagnostic applications is the lack of throughput of plasma/biological fluids nanovesicle isolation. Despite a number of commercially available assays for exosome isolation providing fast protocols, a suitable yield still requires ultracentrifugation protocols of large volumes [22,30]. This greatly limits high-throughput implementation of exosome-based diagnostic approaches. In addition, the specificity and sensitivity in plasma and urine can be reduced by immunoglobulin interference.

\section{Exosomes: novel biomarkers for neurodegenerative diseases?}

Most of the misfolded proteins associated with neurodegeneration are shuttled via exosomes. Superoxide dismutase, $\alpha$-synuclein, amyloid and tau are involved in amyotrophic lateral sclerosis, Parkinson's disease, Alzheimer's disease (AD) and tauopathies, respectively, are all processed via the MVB pathway and secreted in association with exosomes by cultured cells [38-40]. Exosome-associated tau is also present in human cerebrospinal fluid samples of early AD patients [41].

A recent theory suggests that misfolded proteins involved in neurodegenerative diseases can propagate the 'misfolded status' to unaffected brain areas by a prion-like mechanism [42]. The spreading of the newly formed toxic proteins 
within the CNS possibly occurs via the exosomal shuttling system. Exosomes released from $\alpha$-synuclein overexpressing SH-SY5Y cells contain $\alpha$-synuclein. These exosomes can efficiently transfer the protein to normal SH-SY5Y cells [43]. Exosome-associated $\alpha$-synuclein oligomers are more likely to be taken up by recipient cells and can induce more toxicity compared with free oligomers [44]. Another toxic form of synuclein, $\gamma$-synuclein, is also secreted from neuronal cells into conditioned medium in the form of exosomes. It can be transmitted to target cells and cause protein aggregation [45]. Furthermore, plasma exosomes containing amyloidogenic protein oligomers transfer the same type of amyloidosis to other mice via a prion-like mechanism [46]. The 'prion-like' novel concept has crucial implications in understanding the propagation of brain lesions and the progression of amyloid diseases, and highlights the potential of exosomal applications in neurodegenerative medicine.

Interestingly, miRNAs are deregulated in brain tissue during neurodegenerative processes. Significantly different levels of several miRNAs have been found in the AD brain (miR-101, miR-15a, miR-106b, miR-19b, miR-107, miR-298 and miR-328) and cerebrospinal fluid samples (miR-9 and miR-132). In Huntington's disease and in two different Huntington's disease murine models, specific miRNAs, including miR29 and mi-R132 are deregulated in the brain. The same miRNA alterations were also found in the brain of Parkinson's disease patients [47]. Recently miR-206 was identified as a modifier of amyotrophic lateral sclerosis pathogenesis and its role in reinnervating the neuromuscular junction after injury was shown, leading to improved survival in a murine model of the disease [48]. It is currently unknown if these neurodegeneration-associated miRNAs are secreted in exosomes and circulating vesicles. If they are, they could be used as biomarkers for neurodegenerative conditions. Isolation and analysis of neuronal exosomes of patient body fluids is certainly of priority in the field.

\section{Elusive detection of prions in the blood}

Prion diseases (also called transmissible spongiform encephalopathies [TSEs]) are a group of fatal neurodegenerative infectious pathologies, including the human Creutzfeldt-Jakob disease (CJD). They are characterized by the abnormal brain deposition of an insoluble and proteaseresistant form of the prion protein $(\mathrm{PrP})$, named $\mathrm{PrP}^{\mathrm{Sc}}$, which leads to neuronal loss and brain spongiosis [49]. The spread of the disease can also occur via blood transmission [50,51] and as there is no available blood diagnostic test, iatrogenic transmission of CJD is one of the most relevant concerns for public health. The many attempts to develop a proper prion diagnostic test have so far failed, mainly because they have been focused on the detection of the only available marker, $\mathrm{PrP}^{\mathrm{Sc}}$, which has remarkably low levels in blood. The amplification of the misfolded protein via cycles of sonication has provided a number of encouraging assays [52,53] and it was recently demonstrated that certain metal matrices can selectively bind blood disease-related PrP [54]. Nevertheless, a reproducible blood assay with sufficient sensitivity and sensibility for the TSE agent detection is at present still unavailable.

A limiting factor in implementing a successful strategy is the lack of knowledge on how the TSE agent is transported in body fluids. A number of in vitro studies have suggested that the spread of infectivity in between cells and across different tissues and body fluids could also occur via the exosomal pathway. Prions are indeed released from infected murine $\mathrm{N} 2 \mathrm{a}$ and RK13 cells in association with exosomes [55,56]. Interestingly, moloney murine leukemia virus infection strongly enhances the release of prion infectivity in the supernatant of coinfected cells via exosomal recruitment [57]. Hematopoietic cells secrete exosome-associated prions with possible crucial implications in the spreading across the body [58].

In addition, cellular $\operatorname{PrP}\left(\operatorname{Pr} \mathrm{P}^{\mathrm{C}}\right)$ was found to be released by cultured platelets in association with exosomes [59]. These cells express the highest levels of $\mathrm{PrP}^{\mathrm{C}}$ when compared with other blood cells and, indeed, they are an efficient substrate for the in vitro amplification of the abnormal PrP associated with variant CJD [60]. Exosomes released by macrophages into the extracellular space [61] also contain PrPC ${ }^{C}$ where it was shown to bind Hsp70 [61]. $\operatorname{PrP}^{\mathrm{C}}$ was also found in exosomes of cortical neurons [62]. These results are consistent with the intracellular trafficking of $\mathrm{PrP}^{\mathrm{C}}$, known to be a glycosylphosphatidylinositol-anchored protein associated with lipid raft domains and internalized via endocytosis and MVBs [63]. If exosomes are the vehicles of prion infectivity, their isolation in blood could provide the basis for a novel diagnostic approach. In addition, elucidating the mechanisms by which prions spread through the host may help the identification of efficient infection control strategies.

A key issue for the development of a TSE diagnostic assay is the identification of novel reliable markers other than the misfolded protein. 
While the detection of pathological PrP forms in blood remains elusive, the relationship of $\mathrm{PrP}^{\mathrm{Sc}}$ with the TSE infectious agent is currently still unclear. Some data have unequivocally shown that prion infection is not necessarily related to $\mathrm{PrP}^{\mathrm{Sc}}$ as originally described [64-66]. It is therefore crucial to find novel reliable diagnostic markers other than $\mathrm{PrP}^{\mathrm{Sc}}$, such as small RNAs.

It is known that some miRNAs are deregulated in brain tissue and upon prion infection; nevertheless, it is currently unknown if this miRNA species is carried by circulating exosomes.

Specifically, in the brains of mice infected with mouse-adapted scrapie, miRNA profiling showed deregulation of miR-342-3p, miR-320, let-7b, miR-328, miR-128, miR-139-5p and miR-146a, which were over 2.5-fold upregulated, and miR-338-3p and miR-337-3p, which were over 2.5-fold downregulated [67]. Similarly, bovine spongiform encephalopathy-infected macaque brain has significant upregulation of miR-342-3p compared with noninfected animals. miR-342-3p upregulation also occurs in the brain of human type 1 and 2 sporadic CJD patients [68]. Interestingly, exosomes from neuronal cell cultures contain some of these miRNA species that are also deregulated upon prion infection. let-7b, let-7i, miR-128a, miR-21, miR-222, miR-29b, miR-342-3p and miR-424 levels are increased after cell exposure to prions, while miR-146a is reduced [69]. This implies that deregulated miRNA could also be released in association with exosomes in vivo, in the CNS and body fluids. It is crucial to validate this hypothesis in the future with large-scale in vivo studies and ascertain the potential of exosomal miRNAs in the diagnosis of prion diseases.

\section{Exosome \& other human pathological conditions}

The remarkable finding that exosomes contain functional nucleic acids suggests that exosomes resemble viral-like particles [70,71].

Interestingly, many retroviruses, including HIV, have been found to exploit the MVB machinery for propagation [72,73].

Other type of virions such as Epstein-Barr virus (EBV) use the exosomal pathway. For example, EBV miRNAs are secreted by EBV-infected $\mathrm{B}$ cells through exosomes [74]. Interestingly, these exosomal miRNAs can be internalized by dendridic cells where they produce a sound silencing of specific EBV target immunoregulatory genes, therefore favoring the spread of the virus [74].

Human intestinal epithelial cells infected with rotavirus (RV) release exosomes that contain both viral proteins (VP6), and immunomodulators such as heat shock proteins and TGF- $\beta 1$ [75].

The same authors analyzed exosomal preparations from stool samples of 36 children with different forms of gastroenteritis, due or not due to RV. VP6 coimmunoprecipitated with CD63 in most of the RV-infected samples, suggesting that these vesicles are released by RV-infected cells in vivo. Moreover, exosomal fractions from RV-infected cells induced death and inhibited proliferation of $\mathrm{CD}^{+} \mathrm{T}$ cells to a greater extent than fractions from noninfected cells, suggesting that exosomes released upon RV infection can modulate viral immunity [75]. All these findings are in line with the 'Trojan exosome hypothesis' proposed by Gould et al. in 2003 [76], suggesting that exosomes could function as virus Trojan horses, critically contributing to the spread of pathogens in the host upon infection.

Exosomes have an important involvement in kidney-related diseases. Transcription factors such as urinary cAMP-dependent transcription factor 3 and Wilms' tumor protein 1, which are undetectable in whole urine, could be detected in urine-derived exosomes in renal tubular cell injury and podocyte injury-associated chronic kidney disease, respectively [77]. In this latter disease, an early diagnostic sensitive method is still missing, and exosome research is one of the most promising approaches.

In addition, upon nephrotoxic damage, exosomal levels of fetuin-A rapidly increase, significantly before well-established biomarkers such as urine creatinine [77].

Urinary exosomes are also of use in the diagnosis of Bartter syndrome type I, as they do not carry the sodium-potassium-chloride cotransporter, NKCC2 [78]

Interestingly, aquaporin-1 levels in exosomes are reduced for up to 4 days in a rat model of renal ischemia reperfusion and in a person, following renal transplantation [20]. All these studies reflect the great potential of exosome-based methods in the diagnosis and prognosis of kidney-related diseases, but underlie the need for large-scale studies with consistent methodologies.

Further blinded studies are required to validate exosome-based diagnostic methods at preclinical, early- and late-disease onset, storage and processing, and the protocols for protein biomarker identification such as western blot analysis and FACS analysis.

\section{Future perspective}

Available data on exosomes strongly suggest that these nanovesicles may represent the future of 
biomarkers in medicine, as they are the most biomimetic nanovectors for a variety of molecules, including proteins, nucleic acids and chemicals. Exosome-encapsulated curcumin, delivered by the intranasal route is efficient in preventing brain inflammation [79], and specific genesilencing miRNAs enclosed in targeted exosomes and delivered systemically have shown promising therapeutic effects in a mouse AD model [80].

Exosomes may, at the same time, contain disease biomarkers or be the vectors of potential therapeutic molecules, thus representing the ideal theranostic approach [81-83]. This new multidisciplinary field focuses on building nanosystems for future applications of diagnosis and therapy jointly. The theranostic 'all-in-one approach' has a very high potential in the field of personalized medicine, as it allows the detection and monitoring of a disease in individual patients possibly in early clinical stages, as well as targeted drug delivery at the site of the disease.
Exosomes appear to be the ideal vector for this new approach, with the maximal potential of targeting the diseased areas and only minimal side effects.

\section{Acknowledgements}

The authors would like to thank T Azzarito and I Cristofaro for their support in the completion of the table and bibliographical research.

\section{Financial \& competing interests disclosure}

This work was supported by the funds of the Italian Ministry of Health. All authors are employed by the Istituto Superiore di Sanità (Italian National Institute of Health), Rome, Italy. The authors have no other relevant affiliations or financial involvement with any organization or entity with a financial interest in or financial conflict with the subject matter or materials discussed in the manuscript apart from those disclosed.

No writing assistance was utilized in the production of this manuscript.

\section{Executive summary}

\section{Background}

- Exosomes are small vesicles secreted into the extracellular environment upon endosome fusion with the plasma membrane.

- The molecular content of exosomes released into the body fluid is a precious diagnostic tool.

\section{Exosomes \& tumor diagnosis}

- Exosome-associated diagnostic molecules include exosomal proteins and RNAs.

- A number of publications describe the association of exosomal markers with several cancer types; nevertheless, only a few exosome-based diagnostic assays are currently available for clinical use.

- ExoTESTTM (HansaBioMed, Estonia) allows sensitive detection and quantification of exosomes purified from human tumor cell culture supernatant and plasma from severe combined immunodeficiency mice engrafted with a human melanoma based on exosomal protein markers.

- Incorporating ExOTEST technology into a platform for exosome-associated mRNA analysis is expected to enable detection and quantification of plasma RNAs of well-defined tumor origin, providing highly sensitive and specific assessment, while avoiding some of the 'noise' that hampers real-time reverse-transcription PCR and microarray analysis of whole body fluids.

\section{Exosomes \& neurodegenerative diseases}

- Most of the misfolded proteins associated with neurodegeneration are shuttled via exosomes.

- According to the 'prion-like' theory, neurotoxic misfolded proteins are generally transmissible. Their spread within the CNS probably occurs via the exosomal shuttling system.

- miRNAs are deregulated in the CNS during neurodegeneration, but it is currently unknown if they are released in exosome body fluids.

- More research is needed to ascertain the diagnostic value of exosomes in neurodegenerative diseases.

\section{Diagnosis of prion diseases}

- An early diagnostic test for transmissible spongiform encephalopathies is currently unavailable and blood carriers of prions are unknown.

- Prions are shuttled via exosomes in a number of in vitro cell models, but it is currently unknown if this is also occurring in body fluids.

- Some miRNAs are specifically deregulated in the brain of a number of transmissible spongiform encephalopathy models.

- Upon prion infection, these miRNAs are secreted in the extracellular environment in exosomes by cultured cells, suggesting that they might also be released in exosomes into body fluids in vivo.

\section{Exosomes as biomarkers for other pathologies}

- Exosomes have a role in the spread of some types of viruses such as Epstein-Barr virus and rotavirus.

- They are potential biomarkers for kidney-related diseases.

\section{Future perspective}

- Exosomes may, at the same time, contain disease biomarkers or be vectors of potential therapeutic molecules, thus representing the ideal nanotheranostic approach, with a very high potential in the field of personalized medicine. 


\section{References}

Papers of special note have been highlighted as:

- of interest

" " of considerable interest

1 Théry C, Ostrowski M, Segura E. Membrane vesicles as conveyors of immune responses. Nat. Rev. Immunol. 9(8), 581-593 (2009).

- - Recent and complete review on exosome functions.

2 Huotari J, Helenius A. Endosome maturation. EMBO J. 30(17), 3481-3500 (2011).

3 Bobrie A, Colombo M, Raposo G, Théry C. Exosome secretion: molecular mechanisms and roles in immune responses. Traffic 12(12), 1659-1668 (2011).

4 van der Pol E, Böing AN, Harrison P, Sturk A, Nieuwland R. Classification, functions, and clinical relevance of extracellular vesicles. Pharmacol. Rev. 64(3), 676-705 (2012).

5 Heijnen HF, Schiel AE, Fijnheer R, Geuze HJ, Sixma JJ. Activated platelets release two types of membrane vesicles: microvesicles by surface shedding and exosomes derived from exocytosis of multivesicular bodies and $\alpha$-granules. Blood 94(11), 3791-3799 (1999).

6 Johnstone RM, Bianchini A, Teng K. Reticulocyte maturation and exosome release: transferrin receptor containing exosomes shows multiple plasma membrane functions. Blood 74(5), 1844-1851 (1989).

7 Johnstone RM. Exosomes biological significance: a concise review. Blood Cells Mol. Dis. 36(2), 315-321 (2006).

8 Muntasell A, Berger AC, Roche PA. T cellinduced secretion of MHC class II-peptide complexes on B cell exosomes. EMBO J. 26(19), 4263-4272 (2007).

9 Quah BJ, O'Neill HC. The immunogenicity of dendritic cell-derived exosomes. Blood Cells Mol. Dis. 35(2), 94-110 (2005).

10 Simpson RJ, Lim JW, Moritz RL, Mathivanan S. Exosomes: proteomic insights and diagnostic potential. Expert Rev. Proteomics 6(3), 267-283 (2009).

11 Zitvogel L, Regnault A, Lozier A et al. Eradication of established murine tumors using a novel cell-free vaccine: dendritic cell-derived exosomes. Nat. Med. 4(5), 594-600 (1998).

12 Viaud S, Théry C, Ploix S et al. Dendritic cellderived exosomes for cancer immunotherapy: what's next? Cancer Res. 70(4), 1281-1285 (2010).

13 Valadi H, Ekström K, Bossios A, Sjöstrand M, Lee JJ, Lötvall JO. Exosome-mediated transfer of mRNAs and microRNAs is a novel mechanism of genetic exchange between cells. Nat. Cell. Biol. 9(6), 654-659 (2007).

- A milestone in exosome research, showing the transfer of functional RNAs via nanovesicles.
14 Lotvall J, Valadi H. Cell to cell signalling via exosomes through esRNA. Cell Adh. Migr. 1(3), 156-158 (2007).

15 Kosaka N, Iguchi H, Yoshioka Y, Takeshita F, Matsuki Y, Ochiya T. Secretory mechanisms and intercellular transfer of microRNAs in living cells. J. Biol. Chem. 285(23), 17442-17452 (2010).

16 Simpson RJ, Jensen SS, Lim JW. Proteomic profiling of exosomes: current perspectives. Proteomics 8(19), 4083-4099 (2008).

17 Street JM, Birkhoff W, Menzies RI, Webb DJ, Bailey MA, Dear JW. Exosomal transmission of functional aquaporin 2 in kidney cortical collecting duct cells. J. Physiol. (Lond.) 589(Pt 24), 6119-6127 (2011).

18 Nilsson J, Skog J, Nordstrand A et al. Prostate cancer-derived urine exosomes: a novel approach to biomarkers for prostate cancer. Br. J. Cancer 100(10), 1603-1607 (2009).

19 Takata K, Matsuzaki T, Tajika Y, Ablimit A, Hasegawa T. Localization and trafficking of aquaporin 2 in the kidney. Histochem. Cell. Biol. 130(2), 197-209 (2008).

20 Sonoda H, Yokota-Ikeda N, Oshikawa S et al. Decreased abundance of urinary exosomal aquaporin-1 in renal ischemia-reperfusion injury. Am. J. Physiol. Renal Physiol. 297(4), F1006-F1016 (2009).

21 Looze C, Yui D, Leung L et al. Proteomic profiling of human plasma exosomes identifies PPAR $\gamma$ as an exosome-associated protein. Biochem. Biophys. Res. Commun. 378(3), 433-438 (2009).

22 Logozzi M, De Milito A, Lugini L et al. High levels of exosomes expressing CD63 and caveolin-1 in plasma of melanoma patients. PLoS ONE 4(4), e5219 (2009).

w- First clinical evidence of exosomes as biomarkers for melanoma.

23 Rabinowits G, Gerçel-Taylor C, Day JM, Taylor DD, Kloecker GH. Exosomal microRNA: a diagnostic marker for lung cancer. Clin. Lung Cancer 10(1), 42-46 (2009).

24 Michael A, Bajracharya S, Yuen P et al. Exosomes from human saliva as a source of microRNA biomarkers. Oral Dis. 16(1), 34-38 (2010).

25 Palanisamy V, Sharma S, Deshpande A, Zhou H, Gimzewski J, Wong DT. Nanostructural and transcriptomic analyses of human saliva derived exosomes. PLoS ONE 5(1), e8577 (2010).

26 Keller S, Rupp C, Stoeck A et al. CD24 is a marker of exosomes secreted into urine and amniotic fluid. Kidney Int. 72(9), 1095-1102 (2007).

27 Mathivanan S, Ji H, Simpson RJ. Exosomes: extracellular organelles important in intercellular communication. J. Proteomics 73(10), 1907-1920 (2010).

28 Mathivanan S, Lim JWE, Tauro BJ, Ji H, Moritz RL, Simpson RJ. Proteomics analysis of A33 immunoaffinity-purified exosomes released from the human colon tumor cell line LIM1215 reveals a tissue-specific protein signature. Mol. Cell. Proteomics 9(2), 197-208 (2010).

29 Coren LV, Shatzer T, Ott DE. CD 45 immunoaffinity depletion of vesicles from Jurkat $\mathrm{T}$ cells demonstrates that exosomes contain CD 45: no evidence for a distinct exosome/HIV-1 budding pathway. Retrovirology 5, 64 (2008).

30 Keller S, König AK, Marmé F et al. Systemic presence and tumor-growth promoting effect of ovarian carcinoma released exosomes. Cancer Lett. 278(1), 73-81 (2009).

31 Mitchell PJ, Welton J, Staffurth J et al. Can urinary exosomes act as treatment response markers in prostate cancer? J. Transl. Med. 7, 4 (2009).

$32 \mathrm{Lu}$ Q, Zhang J, Allison R et al. Identification of extracellular $\delta$-catenin accumulation for prostate cancer detection. Prostate 69(4), 411-418 (2009).

33 Smalley DM, Sheman NE, Nelson K, Theodorescu D. Isolation and identification of potential urinary microparticle biomarkers of bladder cancer. J. Proteome Res. 7(5), 2088-2096 (2008).

34 Keller S, Ridinger J, Rupp AK, Janssen JWG, Altevogt P. Body fluid derived exosomes as a novel template for clinical diagnostics. J. Transl. Med. 9, 86 (2011).

35 Skog J, Würdinger T, Van Rijn S et al. Glioblastoma microvesicles transport RNA and proteins that promote tumour growth and provide diagnostic biomarkers. Nat. Cell. Biol. 10(12), 1470-1476 (2008).

36 Taylor DD, Gercel-Taylor C. MicroRNA signatures of tumor-derived exosomes as diagnostic biomarkers of ovarian cancer. Gynecol. Oncol. 110(1), 13-21 (2008).

37 Rabinowits G, Gerçel-Taylor C, Day JM, Taylor DD, Kloecker GH. Exosomal microRNA: a diagnostic marker for lung cancer. Clin. Lung Cancer 10(1), 42-46 (2009).

38 Gomes C, Keller S, Altevogt P, Costa J. Evidence for secretion of $\mathrm{Cu}, \mathrm{Zn}$ superoxide dismutase via exosomes from a cell model of amyotrophic lateral sclerosis. Neurosci. Lett. 428(1), 43-46 (2007).

39 Emmanouilidou E, Melachroinou K, Roumeliotis $\mathrm{T}$ et al. Cell-produced $\alpha$-synuclein is secreted in a calciumdependent manner by exosomes and impacts neuronal survival. J. Neurosci. 30(20), 6838-6851 (2010). 
40 Rajendran L, Honsho M, Zahn TR et al. Alzheimer's disease $\beta$-amyloid peptides are released in association with exosomes. Proc. Natl Acad. Sci. USA 103(30), 11172-11177 (2006).

- First in vitro evidence that exosomes are the shuttles of amyloid peptides involved in Alzheimer's disease.

41 Saman S, Kim W, Raya M et al. Exosomeassociated tau is secreted in tauopathy models and is selectively phosphorylated in cerebrospinal fluid in early Alzheimer disease. J. Biol. Chem. 287(6), 3842-3849 (2012).

42 Goedert M, Clavaguera F, Tolnay M. The propagation of prion-like protein inclusions in neurodegenerative diseases. Trends Neurosci. 33(7), 317-325 (2010).

43 Alvarez-Erviti L, Seow Y, Schapira AH et al. Lysosomal dysfunction increases exosomemediated $\alpha$-synuclein release and transmission. Neurobiol. Dis. 42(3), 360-367 (2011).

44 Danzer KM, Kranich LR, Ruf WP et al. Exosomal cell-to-cell transmission of $\alpha$ synuclein oligomers. Mol. Neurodegener. 7, 42 (2012).

45 Surgucheva I, Sharov VS, Surguchov A. $\gamma$-synuclein: seeding of $\alpha$-synuclein aggregation and transmission between cells. Biochemistry 51(23), 4743-4754 (2012).

46 Tasaki M, Ueda M, Ochiai S et al. Transmission of circulating cell-free AA amyloid oligomers in exosomes vectors via a prion-like mechanism. Biochem. Biophys. Res. Commun. 400(4), 559-562 (2010).

47 De Smaele E, Ferretti E, Gulino A. MicroRNAs as biomarkers for CNS cancer and other disorders. Brain Res. 1338, 100-111 (2010).

48 Williams AH, Valdez G, Moresi V et al. MicroRNA-206 delays ALS progression and promotes regeneration of neuromuscular synapses in mice. Science 326(5959), 1549-1554 (2009).

49 Soto C. Diagnosing prion diseases: needs, challenges and hopes. Nat. Rev. Microbiol. 2(10), 809-819 (2004).

50 Aguzzi A, Glatzel M. Prion infections, blood and transfusions. Nat. Clin. Pract. Neurol. 2(6), 321-329 (2006).

51 Houston F, McCutcheon S, Goldmann W et al. Prion diseases are efficiently transmitted by blood transfusion in sheep. Blood 112(12), 4739-4745 (2008).

52 Morales R, Duran-Aniotz C, Diaz-Espinoza $\mathrm{R}$, Camacho MV, Soto C. Protein misfolding cyclic amplification of infectious prions. Nat. Protoc. 7(7), 1397-1409 (2012).

53 McGuire LI, Peden AH, Orrú CD et al. Real time quaking-induced conversion analysis of cerebrospinal fluid in sporadic CreutzfeldtJakob disease. Ann. Neurol. 72(2), 278-285 (2012).

54 Edgeworth JA, Farmer M, Sicilia A et al. Detection of prion infection in variant Creutzfeldt-Jakob disease: a blood-based assay. Lancet 377(9764), 487-493 (2011).

55 Fevrier B, Vilette D, Archer F et al. Cells release prions in association with exosomes. Proc. Natl Acad. Sci. USA 101(26), 9683-9688 (2004).

-1- First evidence of exosomes as prion carriers.

56 Alais S, Simoes S, Baas D et al. Mouse neuroblastoma cells release prion infectivity associated with exosomal vesicles. Biol. Cell 100(10), 603-615 (2008).

57 Leblanc P, Alais S, Porto-Carreiro I et al. Retrovirus infection strongly enhances scrapie infectivity release in cell culture. EMBO J. 25(12), 2674-2685 (2006).

58 Castro-Seoane R, Hummerich H, Sweeting T et al. Plasmacytoid dendritic cells sequester high prion titres at early stages of prion infection. PLoS Pathog. 8(2), e1002538 (2012).

59 Robertson C, Booth SA, Beniac DR, Coulthart MB, Booth TF, McNicol A. Cellular prion protein is released on exosomes from activated platelets. Blood 107(10), 3907-3911 (2006).

60 Jones M, Peden AH, Yull H et al. Human platelets as a substrate source for the in vitro amplification of the abnormal prion protein $(\mathrm{PrP})$ associated with variant Creutzfeldt-Jakob disease. Transfusion 49(2), 376-384 (2009).

61 Wang GH, Zhou XM, Bai Y, Yin XM, Yang LF, Zhao D. Hsp70 binds to $\mathrm{PrP}^{\mathrm{C}}$ in the process of $\mathrm{PrP}^{\mathrm{C}}$ release via exosomes from THP-1 monocytes. Cell Biol. Int. 35(6), 553-558 (2011).

62 Fauré J, Lachenal G, Court $\mathrm{M}$ et al. Exosomes are released by cultured cortical neurones. Mol. Cell. Neurosci. 31(4), 642-648 (2006).

63 Taylor DR, Hooper NM. The prion protein and lipid rafts. Mol. Membr. Biol. 23(1), 89-99 (2006).

64 Lasmézas CI, Deslys JP, Robain O et al. Transmission of the BSE agent to mice in the absence of detectable abnormal prion protein. Science 275(5298), 402-405 (1997).

65 Barron RM, Campbell SL, King D et al. High titers of transmissible spongiform encephalopathy infectivity associated with extremely low levels of $\mathrm{PrP}^{\mathrm{Sc}}$ in vivo. J. Biol. Chem. 282(49), 35878-35886 (2007).

66 Piccardo P, Manson JC, King D, Ghetti B, Barron RM. Accumulation of prion protein in the brain that is not associated with transmissible disease. Proc. Natl Acad. Sci. USA 104(11), 4712-4717 (2007).

67 Saba R, Goodman CD, Huzarewich RL, Robertson C, Booth SA. A miRNA signature of prion induced neurodegeneration. PLoS ONE 3(11), e3652 (2008).

68 Montag J, Hitt R, Opitz L, Schulz-Schaeffer WJ, Hunsmann G, Motzkus D. Upregulation of miRNA hsa-miR-342-3p in experimental and idiopathic prion disease. Mol. Neurodegener. 4, 36 (2009).

69 Bellingham SA, Coleman BM, Hill AF. Small RNA deep sequencing reveals a distinct miRNA signature released in exosomes from prion-infected neuronal cells. Nucleic Acids Res. 40(21), 10937-10949 (2012).

70 Izquierdo-Useros N, Puertas MC, Borràs FE, Blanco J, Martinez-Picado J. Exosomes and retroviruses: the chicken or the egg? Cell. Microbiol. 13(1), 10-17 (2011).

71 Izquierdo-Useros N, Naranjo-Gómez M, Archer J et al. Capture and transfer of HIV-1 particles by mature dendritic cells converges with the exosome-dissemination pathway. Blood 113(12), 2732-2741 (2009).

72 De Gassart A, Trentin B, Martin M et al. Exosomal sorting of the cytoplasmic domain of bovine leukemia virus TM Env protein. Cell. Biol. Int. 33(1), 36-48 (2009).

73 Gan X, Gould SJ. Identification of an inhibitory budding signal that blocks the release of HIV particles and exosome/microvesicle proteins. Mol. Biol. Cell 22(6), 817-830 (2011).

74 Pegtel DM, Cosmopoulos K, Thorley-Lawson DA et al. Functional delivery of viral miRNAs via exosomes. Proc. Natl Acad. Sci. USA 107(14), 6328-6333 (2010).

75 Barreto A, Rodríguez LS, Rojas OL et al. Membrane vesicles released by intestinal epithelial cells infected with rotavirus inhibit T-cell function. Viral Immunol. 23(6), 595-608 (2010).

76 Gould SJ, Booth AM, Hildreth JEK. The trojan exosome hypothesis. Proc. Natl Acad. Sci. USA 100(19), 10592-10597 (2003).

77 Zhou H, Pisitkun T, Aponte A et al. Exosomal Fetuin-A identified by proteomics: a novel urinary biomarker for detecting acute kidney injury. Kidney Int. 70(10), 1847-1857 (2006).

78 Gonzales PA, Pisitkun T, Hoffert JD et al. Large-scale proteomics and phosphoproteomics of urinary exosomes. J. Am. Soc. Nephrol. 20(2), 363-379 (2009).

79 Zhuang X, Xiang X, Grizzle W et al. Treatment of brain inflammatory diseases by delivering exosome encapsulated antiinflammatory drugs from the nasal region to the brain. Mol. Ther. 19(10), 1769-1779 (2011). 
80 Alvarez-Erviti L, Seow Y, Yin H, Betts C, Lakhal S, Wood MJA. Delivery of siRNA to the mouse brain by systemic injection of targeted exosomes. Nat. Biotechnol. 29(4), 341-345 (2011).

- Elegant demonstration that exosomes could be used as vectors of therapeutic drugs for treating Alzheimer's disease.

81 Hood JL, Wickline SA. A systematic approach to exosome-based translational nanomedicine. Wiley Interdiscip. Rev. Nanomed. Nanobiotechnol. 4(4), 458-467 (2012).

82 Vlassov AV, Magdaleno S, Setterquist R, Conrad R. Exosomes: current knowledge of their composition, biological functions, and diagnostic and therapeutic potentials. Biochim. Biophys. Acta 1820(7), 940-948 (2012).

83 van Dommelen SM, Vader P, Lakhal S et al. Microvesicles and exosomes: opportunities for cell-derived membrane vesicles in drug delivery. J. Control. Release 161(2), 635-644 (2012).

84 Rood IM, Deegens JK, Merchant ML et al. Comparison of three methods for isolation of urinary microvesicles to identify biomarkers of nephrotic syndrome. Kidney Int. 78(8), 810-816 (2010).

85 Wittmann J, Jäck HM. Serum microRNAs as powerful cancer biomarkers. Biochim. Biophys. Acta 1806(2), 200-207 (2010).
Corcoran C, Friel AM, Duffy MJ, Crown J, O'Driscoll L. Intracellular and extracellular microRNAs in breast cancer. Clin. Chem. 57(1), 18-32 (2011).

87 Li J, Sherman-Baust CA, Tsai-Turton M, Bristow RE, Roden RB, Morin PJ. Claudincontaining exosomes in the peripheral circulation of women with ovarian cancer. BMC Cancer 9, 244 (2009).

88 Bala S, Petrasek J, Mundkur S et al. Circulating microRNAs in exosomes indicate hepatocyte injury and inflammation in alcoholic, drug-induced, and inflammatory liver diseases. Hepatology 56(5), 1946-1957 (2012). 